\title{
Uso de recubrimientos a base de nanoemulsión como alternativa para conservar la calidad poscosecha del jitomate.
}

\section{Use of coating with nanoemulsion as alternative of conservation the postharvest quality of tomato.}

\author{
Kenia I. Badillo-Solis ${ }^{a}$, Elizabeth Pérez-Soto ${ }^{a}$, Gabriel Aguirre-Álvarez $^{a}$, Juan Ocampo-López \\ ${ }^{a}$, Antonio de J. Cenobio-Galindo ${ }^{a}$, Rafael G. Campos-Montiel ${ }^{a, b}$.
}

\begin{abstract}
:
In this time the food industries are looking a new technology to increase the shelf life of fresh products. The food coatings are economic technology and friendly with environment. The layers are development with lipids, polysaccharides and proteins to have a barrier to steam water, gases and other solutes. The use of nanoemulsions as coating is an excellent system to release functional ingredients as antimicrobial and antioxidant agent to improve to shelf life of fruits and fresh vegetables or with minimal process.
\end{abstract}

Keywords:

Coating, nanoemulsion, bioactive compounds, tomato.

\section{Resumen:}

Hoy en día la industria alimentaria busca nuevas tecnologías que permitan aumentar la vida útil de productos frescos. Los recubrimientos alimenticios son una tecnología económica y amigable con el medio ambiente, dichas capas de recubrimiento pueden desarrollarse de lípidos, polisacáridos y proteínas funcionando como barreras contra el vapor de agua, los gases, y otros solutos. El uso de nanoemulsiones como recubrimiento proporciona un excelente sistema de liberación de ingredientes funcionales, como los agentes antimicrobianos y antioxidantes, mejorando así la calidad y extendiendo la vida útil de frutas y hortalizas frescas o mínimamente procesadas.

\section{Palabras Clave:}

Recubrimiento, nanoemulsión, compuestos bioactivos, jitomate.

\section{Introducción}

El jitomate (Solanum lycopersicum L.) es uno de los principales cultivos a nivel mundial ya que además de su importancia económica es también fuente de vitaminas, minerales y antioxidantes importantes para la nutrición humana 1. México es el principal exportador de jitomate en el mundo, siendo el tipo saladette y bola los mayormente exportados 2, con destino a Estados Unidos. Los frutos son climatéricos y durante la vida poscosecha sufren cambios fisiológicos considerables durante su transporte y almacenamiento, principalmente la concentración de etileno y el desarrollo de microorganismos como bacterias y hongos 3 .

Para conservar la calidad de los frutos durante la poscosecha se ha empleado en los últimos años el uso de recubrimientos. Estos forman una fina capa sobre el alimento y actúan como barrera semipermeable a los gases y al vapor de agua, mejoran las propiedades mecánicas, mantienen la integridad estructural del producto y retienen compuestos volátiles 4 .

\footnotetext{
${ }^{a}$ Universidad Autónoma del Estado de Hidalgo, Instituto de Ciencias Agropecuarias, Rancho Universitario, Av.Universidad Km. 1 s/n Exhacienda Aquetzalpa, C.P.43600 Tulancingo de Bravo, Hidalgo. Kenia I. Badillo Solis, ba259539@uaeh.edu.mx, https://orcid.org/0000-0001-8575-8897; Elizabeth Pérez-Soto, epsoto@uaeh.edu.mx; https://orcid.org/0000-0002-5195-6630; Gabriel Aguirre-Álvarez, aguirre@ uaeh.edu.mx, https://orcid.org/0000-0001-7652-2647; Juan Ocampo-López, jocampo@uaeh.edu.mx, https://orcid.org/0000-0002-9208-7216; Antonio de Jesús Cenobio-Galindo, antonio_cenobio@uaeh.edu.mx, https://orcid.org/0000-0003-3098-0487; Rafael G. Campos-Montiel, rcampos@uaeh.edu.mx, https://orcid.org/0000-0001-7382-5538.

${ }^{\mathrm{b}}$ Autor de Correspondencia.
} 
Actualmente, la nanotecnología ha sido introducida con éxito en el área de los alimentos 5. Las nanoemulsiones usadas como recubrimiento alimenticio proporcionan una excelente barrera contra la humedad 6, debido a la incorporación de compuestos bioactivos provenientes principalmente de fuentes vegetales 7 , como frutas y hortalizas.

Los frutos de xoconostle (Opuntia oligacantha), son originarios de la zona central de México. De acuerdo a diversos estudios 8,9 posee compuestos bioactivos tales como polifenoles con propiedades antioxidantes y antimicrobianas.

Así mismo, se han incorporado aceites esenciales en diversos recubrimientos 10 , debido a su actividad antioxidante y antimicrobiana. El aceite esencial de naranja (Citrus sinensis) posee gran cantidad de compuestos bioactivos, debido a la presencia de flavonoides 11 .

\section{Jitomate (Solanum lycopersicum L.)}

El jitomate es uno de los cultivos con mayor demanda en México y en el mundo, debido al contenido de compuestos bioactivos que han mostrado tener posibles efectos en la salud humana 12. Además, es un alimento de escaso contenido calórico ya que $100 \mathrm{~g}$ de jitomate aportan solamente $18 \mathrm{kcal} \mathrm{13}$. Es fuente importante de minerales como el potasio y magnesio, de su contenido de vitaminas destacan la A, B1, B2, y C. Esta última junto con el licopeno (pigmento que otorga el color rojo característico) son antioxidantes importantes para el organismo 14. De acuerdo a Vinha et al. 2014, 12 posee tocoferoles y polifenoles, compuestos que han mostrado tener posibles efectos anticancerígenos, antihipertensivos y antimutagénicos.

México es el principal exportador de jitomate en el mundo, en 2017 participó con el $24 \%$ del total de las exportaciones y ocupa el segundo lugar de los productos agrícolas más exportados 15,16. Actualmente, se satisface el $100 \%$ de los requerimientos nacionales con la producción interna y el $48 \%$ de la disponibilidad total de jitomate es destinado para exportación, siendo enero, febrero, marzo y abril los meses de mayor flujo comercial al extranjero, principalmente con destino a Estados Unidos. El 98\% de las exportaciones de jitomate mexicano corresponden a jitomate saladette y bola y el $2 \%$ restante a jitomate cherry 2.

\section{Métodos de conservación}

Los frutos de jitomate son climatéricos, aumentando la tasa de respiración y producción de etileno durante la maduración. Una vez cosechados, sufren cambios fisiológicos considerables durante su almacenamiento, tales como: pérdida de propiedades organolépticas, descenso de componentes nutricionales y pérdida de humedad que genera el ablandamiento del tejido y por consiguiente su podredumbre 17. Por otra parte, factores como: manipulación inadecuada, humedad, luz solar, temperatura, crecimiento y actividad de microorganismos patógenos afectan la vida útil de los frutos 18 .

De acuerdo a la FAO 19, se estima que a nivel mundial las pérdidas poscosecha de frutas y hortalizas son aproximadamente del $5-25 \%$ en países desarrollados y del 20 al $50 \%$ en países en desarrollo, por tanto, para combatir los diferentes factores que ocasionan el deterioro de frutas y hortalizas se han desarrollado diversos métodos de conservación, entre los que se encuentran el uso de bajas temperaturas, tratamientos químicos, aplicación de atmósferas modificadas o controladas y recubrimientos alimenticios 20 .

\subsection{Recubrimientos alimenticios}

Los recubrimientos alimenticios son definidos como capas delgadas que sirven como barrera entre el alimento y su entorno durante su manejo, procesamiento y almacenamiento 21. De acuerdo con Eissa 22, las frutas y hortalizas son frecuentemente cubiertas al sumergir 0 asperjar un variado número de materiales comestibles entre los que se encuentran lípidos, polisacáridos, y proteínas.

\section{$2.2 \quad$ Tipos de recubrimientos}

Los recubrimientos incorporados en alimentos se componen de materiales biológicos y con base a esto pueden clasificarse en 3 tipos: hidrocoloides, compuestos y lipídicos. Los recubrimientos hidrocoloides son polímeros solubles en agua obtenidos a partir de plantas, animales o fuentes microbianas. Se encuentran en estado puro como el almidón y celulosa, ambos son utilizados para la preparación de películas biodegradables 23 .

Los recubrimientos compuestos proporcionan una buena barrera al vapor de agua y una barrera selectiva para el oxígeno y dióxido de carbono. Estos revestimientos pueden ser una mezcla de polisacáridos, proteínas y/o lípidos 24.

Finalmente, los recubrimientos basados en lípidos han sido utilizados exitosamente en frutas y hortalizas enteras reduciendo la superficie de daño durante el manejo y como barrera ante la pérdida de humedad prolongando su vida útil 6 . Dentro de las sustancias lipídicas más eficaces encontramos a la parafina, ceras, ácidos grasos, aceites esenciales, resinas y surfactantes 25. Un tipo de recubrimiento lipídico, son los sistemas emulsionados, los cuales consisten en dos líquidos inmiscibles (generalmente agua y aceite), en donde uno de ellos es 
dispersado en forma de pequeñas gotas dentro del otro 26.

\section{Nanoemulsiones como recubrimiento}

Las emulsiones con tamaño de partícula entre 20 y 100 $\mathrm{nm}$ son denominadas nanoemulsiones 27 , y han sido introducidas con éxito en el área de los alimentos en los últimos años debido a que presentan ventajas importantes en comparación a las emulsiones convencionales. Dentro de las bondades de las nanoemulsiones como recubrimiento destacan: pequeño tamaño de partícula, transparencia o claridad óptica, alta relación superficievolumen, estabilidad cinética por largos períodos de tiempo y alta disponibilidad oral a los bioactivos dispersos 28. Así mismo, de acuerdo con Choi et al. 29, el uso de nanoemulsiones como recubrimiento en alimentos a mostrado tener un mínimo impacto en las características organolépticas del producto.

\subsection{Métodos de fabricación}

De acuerdo a McClements 30, las emulsiones se clasifican según la distribución de la fase dispersa (sustancia formadora de gotas), teniendo que el sistema en el cual las partículas de aceite se dispersan en una fase acuosa se denomina emulsión aceite en agua $(\mathrm{O} / \mathrm{W})$ por sus siglas en inglés, mientras que aquel en el que las gotas de agua se dispersan en una fase oleosa se conoce como emulsión agua en aceite (W/O).

En la elaboración de nanoemulsiones es necesaria la aplicación de energía para que se unan el compuesto lipídico y el acuoso, ya que esta unión no puede presentarse de manera natural o espontánea. Respecto a la cantidad de energía empleada para su formación, los procesos han sido clasificados en procesos de baja y alta energía. En el primero de ellos encontramos la composición de inversión de fases (PIC) y la temperatura de inversión de fases (PIT). Mientras que los procesos de alta energía son los más utilizados y requieren el uso de dispositivos mecánicos que generan las fuerzas disruptivas necesarias para lograr el rompimiento de las fases macroscópicas en la emulsión 31. De acuerdo a Jafari et al. 32, dentro de esta última categoría encontramos al ultrasonido, micro fluidificación y altas presiones.

\subsection{Incorporación de compuestos bioactivos}

Las nanoemulsiones permiten encapsular compuestos con potencial actividad biológica (carotenoides, vitaminas, polifenoles), además de hacer más viable la incorporación de componentes de naturaleza lipofílica como aceites esenciales, glicéridos y ácidos grasos 33 .

La incorporación de aceites esenciales en recubrimientos alimenticios se ha reportado en manzanas, brócoli y piña 34,35,36. El aceite esencial de naranja (Citrus sinensis) posee gran cantidad de compuestos bioactivos, debido a la presencia de flavonoides 11. Se ha empleado en la elaboración de nanoemulsiones, gracias a sus propiedades antioxidantes y antimicrobianas 37, lo que ha reducido la tasa de respiración y deterioro microbiano de los productos frescos 38 .

Los compuestos bioactivos incorporados en las nanoemulsiones, también provienen de fuentes vegetales como frutas y hortalizas 7. México constituye uno de los centros de diversidad más importantes de cactáceas, siendo el más variado y ampliamente distribuido de América, con alrededor de 191-215 especies 39. Diversos estudios reportan que los frutos ácidos de xoconostle (Opuntia oligacantha) contienen compuestos bioactivos como polifenoles con propiedades antioxidantes y antimicrobianas 8, además de actividad antihipoglucémica, hipocolesterolemiante antihiperlipidémica, antiinflamatoria, antiulcerogénica y en la prevención de algunos tipos de cáncer 40,41. Cenobio-Galindo et al. 42, desarrollaron una nanoemulsión con extracto de xoconostle incorporándola como recubrimiento en aguacate Hass, conservando la calidad poscosecha de los frutos hasta por 60 días.

\subsection{Aplicación de nanoemulsiones como recubrimiento en alimentos}

Actualmente la aplicación de nanoemulsiones como recubrimiento alimenticio es variado. En frutos de tomate cherry, se aplicó un recubrimiento con nanoemulsión de timol incorporada mediante películas comestibles de quinoa y quitosano, el cual inhibió en una concentración de 110 ppm de timol a Botrytis cinerea 43.

Kim et al. 44, recubrieron uvas con nanoemulsiones de cera de carnauba y aceite esencial de limoncillo, preservando así los compuestos fenólicos y la actividad antioxidante de los frutos hasta por 28 días. Además, la concentración del 3\% de aceite esencial de limoncillo, registró una disminución de $>2.8 \mathrm{log} \mathrm{UFC} / \mathrm{g}$ de $E$. coli 0157:H7 y Salmonella typhimurium.

Así mismo, se incorporó un recubrimiento con nanoemulsión de aceite esencial de canela en fresas 45 , reduciendo la pérdida de propiedades fisicoquímicas y mostrando una mayor actividad antimicrobiana contra mohos y bacterias (1.958 y 2.544 log UFC/g, respectivamente) en comparación con los frutos sin recubrimiento. 


\section{Conclusión}

La incorporación de nanoemulsiones como recubrimiento en alimentos mínimamente procesados como el jitomate va en aumento, debido a que son una excelente alternativa para conservar la calidad poscosecha, al reducir la superficie de daño y actuar como una barrera ante la pérdida de humedad, gases y solutos durante el almacenamiento. Estos sistemas permiten la encapsulación y conservación de compuestos bioactivos provenientes principalmente de fuentes vegetales y aceites esenciales, manteniendo así las características organolépticas como textura, color y sabor.

\section{Referencias}

[1] Antunes, M. D., Rodrigues, D., Pantazis, V., Cavaco, A. M., Siomos, A. S., \& Graca, M. (2013). Nutritional Quality Changes of Fresh-cut Tomato during Shelf Life. Food Science and Biotechnology, 22(5), 1229-1236. https://doi.org/10.1007/s10068-013-0206-6

[2] SIAP.Servicio de Información Agroalimentaria y Pesquera. (2018).

[3] Hussen, S. (2014). Ethylene as a Postharvest "Evil" and its Remedies in some Horticultural Crops . Greener Journal of Plant Breeding and Crop Science, 34-40. https://www.researchgate.net/publication/292963213

[4] Navarro, M. (2007). Efecto de la composición de recubrimientos comestibles a base de hidroxipropilmetilcelulosa y cera de abeja en la calidad de ciruelas, naranjas y mandarinas. Tesis doctoral. España: Universidad Politécnica de Valéncia. Departamento de Tecnología de Alimentos.

[5] Ranjan, S., Dasgupta, N., Chakraborty, A. R., \& Melvin, S. (2014). Nanoscience and Nanotechnologies in Food Industries: Opportunities and Research Trends. Journal of Nanoparticle Research, 16(6), 147-152. https://www.researchgate.net/publication/262309141

[6] Baldwin, E., Níspero-Carriedo, M., \& Baker, R. (1995). Use of edible coatings to preserve quality of lightly (and slightly) processed products. Food Sci. Nutr. , 509-524. https://doi.org/10.1080/10408399509527713

[7] Valdés, A., Mellinas, A., Ramos, M., Burgos, N., Jiménez, A., \& Garrigós, M. (2015). Use of herbs, spices and their bioactive compounds in active food packaging. RSC Advances, 5(50), 40324-40335. https://doi.org/10.1039/C4RA17286H

[8] Hernández-Fuentes, A., Trapala-Islas, A., Gallegos-Vásquez, C., Campos-Montiel, R., Pinedo-Espinoza, J., \& Guzmán-Maldonado, S. (2015). Physicochemical variability and nutritional and functional characteristics of xoconostles (Opuntia spp.) accessions from Mexico. Fruits, 70(2), 109-116. https://doi.org/10.1051/fruits/2015002

[9] Espinosa-Muñoz, V., Roldán-Cruz, C., Hernández-Fuentes, A., Quintero-Lira, A., Almaraz-Buendía, I., \& Campos-Montiel, R. (2016). Ultrasonic-Assisted Extraction of Phenols, Flavonoids, and Biocompounds with Inhibitory Effect Against Salmonella Typhimurium and Staphylococcus Aureus from Cactus Pear. Journal of Food Process Engineering, 40(2), e12358. https://doi.org/10.1111/jfpe.12358
[10] Sánchez-González, L., Vargas, M., González-Martínez, C., Chiralt, A., \& Cháfer, M. (2011). Use of essential oils in bioactive adible coatings. Food Engineering Reviews, 1-16. https://doi.org/10.1007/s12393-010$\underline{9031-3}$

[11] Tenorio-Domínguez, M. (2016). Flavonoids extracted from orange peelings tangelo (Citrus reticulata $\mathrm{x}$ Citrus paradisi) and their application as a natural antioxidant in sacha inchi (Plukenetia volubilis) vegetable oil. Scientia Agropecuaria, 7(4), 419-431. https://doi.org/10.17268/sci.agropecu.2016.04.07

[12] Vinha, A. F., Alves, R. C., Barreira, S. V., Castro, A., Costa, A. S., \& Oliveira, M. P. (2014). Effect of peel and seed removal on the nutritional value and antioxidant activity of tomato (Lycopersicon esculentum L.) fruits. LWT - Food Science and Technology, 55, 197-202. http://dx.doi.org/10.1016/j.1wt.2013.07.016

[13] USDA. Departamento de Agricultura de los Estados Unidos. (2017). https://www.usda.gov/

[14] Lobato Ortiz, R., Rodríguez Guzmán, E., Carillo Rodríguez, J., Chávez Servia, J., Sánchez Peña, P., \& Aguilar Meléndez, A. (2012). Exploración, colecta y conservación de recursos genéticos de jitomate: avances en la Red de Jitomate. Sistema Nacional de Recursos Fitogenéticos para la Alimentación y la Agricultura (SINAREFI), 54.

[15] TRADE MAP. Trade statistics for international business development. (2018). https://www.trademap.org/Index.aspx

[16] SAGARPA. Secretaría de Agricultura, Ganadería, Desarrollo Rural, Pesca y Alimentación. https://www.gob.mx/cms/uploads/attachment/file/257077/PotencialJitomate.pdf

[17] Salas-Méndez, E. d., Pinheiro, A. C., Ballesteros, L. F., Silva, P., Rodríguez-García, R., Hernández-Castillo, F. D., . . . de Rodríguez, D. J. (2019). Application of edible nanolaminate coatings with antimicrobial extract of Flourensia cernua to extend the shelf-life of tomato (Solanum lycopersicum L.) fruit. Postharvest Biology and $\begin{array}{lll}\text { Technology, } & 150, & 19-27 .\end{array}$ https://doi.org/10.1016/j.postharvbio.2018.12.008

[18] Mutari, A., \& Debbie, R. (2011). The effects of postharvest handling and storage temperature on the quality and shelf of tomato. African Journal of Food Science , 340-348. https://doi.org/10.5897/AJFS.9000283

[19] FAO. (2017). Reducir pérdidas de alimentos y lograr sistemas alimentarios sostenibles. Recomendaciones de políticas públicas para México. http://www.fao.org/3/i7053s/i7053s.pdf

[20] Ruíz, J., Vicente, A., Montañéz-Saenz, J. C., Rodríguez-Herrera, R., \& Aguilar-González, C. N. (2012). Un tesoro perecedero en México: el tomate, tecnologías para prolongar su vida de anaquel. Investigación y Ciencia: Universidad Autónoma de Aguascalientes, 57-63. http://www.redalyc.org/articulo.oa?id=67424408008

[21] Sharma, P., Shehin, V. P., Kaur, N., \& Vyas, P. (2019). Application of edible coatings on fresh and minimally processed vegetables: a review. International Journal of Vegetable Science., 295-314. https://doi.org/10.1080/19315260.2018.1510863 
[22] Eissa, H. A. (2007). Effect of chitosan coating on shelf life and quality of fresh-cut mushroom. Journal of Food Quality, 623-645. https://www.researchgate.net/publication/229777424

[23] Falguera, V., Quintero, J. P., Jiménez, A., Muñoz, J. A., \& Ibarz, A. (2011). Edible films and coatings: Structures, active functions and trends in their use. Trends in Food Science \& Technology, 22(6), 292303. https://www.researchgate.net/publication/275969518

[24] Dhall, R. (2013). Advances in edible coatings for fresh fruits and vegetables: a review. Critical Reviews in Food Science and Nutrition, 53(5), 435-450. https://doi.org/10.1080/10408398.2010.541568

[25] Hall, D. J. (2011). Edible coatings from lipids, waxes, and resins. En D. J. Hall, Edible Coatings and Films to Improve Food Quality (págs. 79-101). CRC Press.

[26] McClements, D. (2005). Food Emulsions: Principles, Practices, and Techniques. Boca Raton, FL: CRC Press.

[27] Shakeel, F., Talegaonkar, S., Ahmad, F., Khar, R., Ali, M., \& Shafiq, S. (2007). Development and bioavailability assessment of ramipril nanoemulsion formulation. European Journal of Pharmaceutics and Biopharmaceutics, 227-243. https://doi.org/10.1016/j.ejpb.2006.10.014

[28] McClements, D. J. (2012). Nanoemulsions versus microemulsions: terminology, differences, and similarities. Soft Matter, 8(6), 1719-1729. https://doi.org/10.1039/C2SM06903B

[29] Choi, A., Kim, C., Cho, Y., Hwang, J., \& Kim, C. (2011). Characterization of capsaicin-loaded nanoemulsions stabilized with alginate and chitosan by self-assembly. Food and Bioprocess Technology, 4(6), 1119-1126. https://doi.org/10.1007/s11947-011$\underline{0568-9}$

[30] McClements, D. (1999). Food emulsions. Principles, practice and techniques. Florida, EE.UU.: Press LLC.

[31] Abbas, S., Hayat, K., Karanqwa, E., Bashari, M., \& Zhang, X. (2013). An Overview of Ultrasound Assisted Food-Grade Nanoemulsions. Food Engineering Reviews, 5(3), 139-157. https://doi.org/10.1007/s12393013-9066-3

[32] Jafari, S., He, Y., \& Bhandari, B. (2006). Nano-Emulsion Production by Sonication and Microfluidization-A Comparison. International Journal of Food Properties, 9(3), 475-485. https://doi.org/10.1080/10942910600596464

[33] McClements, D. J., \& Rao, J. (2011). Food-Grade Nanoemulsions: Formulation, Fabrication, Properties, Performance, Biological Fate, and Potential Toxicity. Critical Reviews in Food Science and Nutrition, 51:4, 285-330. https://doi.org/10.1080/10408398.2011.559558

[34] Zambrano-Zaragoza, M. L., Gutiérrez-Cortez, E., Del Real, A., González-Reza, R. M., Galindo-Pérez, M. J., \& Quintanar-Guerrero, D. (2014). Fresh-cut Red Delicious apples coating using tocopherol/mucilage nanoemulsion: Effect of coating on polyphenol oxidase and pectin methylesterase activities. Food Research International. $62, \quad 974-983$. https://doi.org/10.1016/j.foodres.2014.05.011

[35] Severino, R., Dang Vu, K., Dons, F., Salmieri, S., Ferrari, G., \& Lacroix, M. (2014). Antimicrobial effects of different combined non- thermal treatments against Listeria monocytogenes in broccoli florets. Journal of Food Engineering, 124, 1-10. https://doi.org/10.1016/j.jfoodeng.2013.09.026

[36] Prakash, A., Baskaran, R., \& Vadivel, V. (2020). Citral nanoemulsion incorporated edible coating to extend the shelf life of fresh cut $\begin{array}{llll}\text { pineapples. } & \text { LWT, } & 118, & 108851 .\end{array}$ https://doi.org/10.1016/j.lwt.2019.108851

[37] Zhang, Z., Vriesekoop, F., Yuan, Q., \& Liang, H. (2014). Effects of nisin on the antimicrobial activity of d-limonene and its nanoemulsion. Food Chemistry, volumen 150, 307-312. https://doi.org/10.1016/j.foodchem.2013.10.160

[38] Saxena, A., Sharma, L., \& Maity, T. (2020). Enrichment of edible coatings and films with plant extracts or essential oils for the preservation of fruits and vegetables. En Biopolymer-Based Formulations (págs. 859-880). https://doi.org/10.1016/B978-0-12$\underline{816897-4.00034-5}$

[39] Gallegos-Vázquez, C., Scheinvar, L., Nuñez-Colín, C. A., \& Mondragón-Jacobo, C. (2012). Morphological diversity of xoconostles (Opuntia spp.) or acidic cactus pears: a Mexican contribution to $\begin{array}{llll}\text { functional foods. } & \text { Fruits, }\end{array}$ https://doi.org/10.1051/fruits/2012001

[40] Guzmán-Maldonado, S. H., Morales-Montelongo, A. L., MondraagónJacobo, C., Herrera-Hernández, M. G., Guevara-Lara, F., \& ReynosoCamacho, R. (2010). Physicochemical, nutritional and functional characterization of fruits xoconostle (Opuntia matudae) pears from central-Mexico region. Journal Food Science, 75, 485-491. https://doi.org/10.1111/j.1750-3841.2010.01679.x

[41] Morales, P., Barros, L., Ramírez-Moreno, E., Santos-Buelga, C., \& Ferreira, I. (2015). Xoconostle fruit (Opuntia matudae Scheinvar cv. Rosa) by-products as potential functional ingredients. Food Chemistry, 185, 289-297. https://doi.org/10.1016/j.foodchem.2015.04.012

[42] Cenobio-Galindo, A. d., Ocampo-López, J., Reyes-Munguía, A., Carrillo-Inungaray, M. L., Cawood, M., Medina-Pérez, G., . . CamposMontiel, R. G. (2019). Influence of Bioactive Compounds Incorporated in a Nanoemulsion as Coating on Avocado Fruits (Persea americana) during Postharvest Storage: Antioxidant Activity, Physicochemical Changes and Structural Evaluation. Antioxidants, 1-12. https://doi.org/10.3390/antiox8100500

[43] Robledo, N., Vera, P., López, L., Mehrdad, Y. P., Tapia, C., \& Abugoch, L. (2018). Thymol nanoemulsions incorporated in quinoa protein/chitosan edible films; antifungal effect in cherry tomatoes. Food Chemistry, 246, 211-219. https://doi.org/10.1016/j.foodchem.2017.11.032

[44] Kim, I.-H., Oh, Y., Lee, H., Song, K., \& Min, S. (2014). Grape berry coatings of lemongrass oil-incorporating nanoemulsion. LWT-Food Science and Technology, 1-10. https://doi.org/10.1016/j.1wt.2014.03.018

[45] Chu, Y., Gao, C., Liu, X., Zhang, N., Xu, T., Feng, X., . . Tang, X. (2020). Improvement of storage quality of strawberries by pullulan coatings incorporated with cinnamon essential oil nanoemulsion. LWT, 122, 109054. https://doi.org/10.1016/j.lwt.2020.109054 